\title{
Reverse mathematics and colorings of hypergraphs
}

\author{
C. Davis, J. Hirst, J. Pardo, and T. Ransom
}

April 5, 2018

\begin{abstract}
Working in subsystems of second order arithmetic, we formulate several representations for hypergraphs. We then prove the equivalence of various vertex coloring theorems to $\mathrm{WKL}_{0}, \mathrm{ACA}_{0}$, and $\Pi_{1}^{1}-\mathrm{CA}_{0}$. MSC: 03B30; 03F35
\end{abstract}

A hypergraph consists of a set of vertices together with a set of edges. While edges in graphs always have exactly two vertices, hypergraphs may have edges of any cardinality, finite or infinite. Some authors exclude edges that are empty of have a single vertex, but for this article, edges may be of any size. After considering alternatives for the representations of edges, we will turn to results on the strength of theorems related to three types of vertex colorings.

\section{Representations of edges}

The edges of hypergraphs can be presented in a variety of ways. If all the edges are finite, each edge can be encoded by a single number. In this case, the edges can be represented by a set of codes or by a sequence of codes. Finite edges and infinite edges can both be represented by a sequence of

${ }^{*}$ Corresponding author: Jeffry Hirst, Department of Mathematical Sciences, Appalachian State University, Boone, NC 28608 hirstjl@math.appstate.edu ORCID:00000002-8273-8951

This is a pre-print of an article published in the Archive for Mathematical Logic. The final authenticated version is available online at: https://doi.org/10.1007/s00153-018-0654 
characteristic functions. Some changes in representation can be carried out in $\mathrm{RCA}_{0}$, while others require additional set comprehension, as shown by the next four results.

Theorem 1. $\left(\mathrm{RCA}_{0}\right)$ If $H$ is a hypergraph with finite edges represented by a set of edges, then there is a hypergraph $H^{\prime}$ with exactly the same edges represented by a sequence of its edges, possibly with repetitions.

Proof. We argue in $\mathrm{RCA}_{0}$. Suppose $H=\langle V, E\rangle$ is a hypergraph with finite edges, where $E$ is a set of integer codes for the edges of $H$. Let $e_{0}$ be an integer code for an edge of $H$. Define the function $f: \mathbb{N} \rightarrow \mathbb{N}$ by

$$
f(n)= \begin{cases}n & n \in E \\ e_{0} & \text { otherwise }\end{cases}
$$

Then the range of $f$ is $E$, and $V$ together with $f$ represents $H$ using a sequence of codes.

Theorem 2. $\left(\mathrm{RCA}_{0}\right)$ If $H$ is a hypergraph with finite edges represented by a sequence of its edges, then there is a hypergraph $H^{\prime}$ with exactly the same edges represented by a sequence of characteristic functions for its edges.

Proof. We argue in $\mathrm{RCA}_{0}$. Suppose $H=\langle V, E\rangle$ is a hypergraph with finite edges, where $E$ is the sequences for the edges of $H$. Define the characteristic function $\chi_{i}$ by

$$
\chi_{i}\left(v_{j}\right)= \begin{cases}1 & v_{j} \in\left\langle e_{i}\right\rangle \\ 0 & \text { otherwise }\end{cases}
$$

By recursive comprehension, the sequence of characteristic function $\left\langle\chi_{i}\right\rangle_{i \in \mathbb{N}}$ exists. The vertices $V$ together with the sequence of characteristic functions for each edge represent $H$.

Theorem 3. $\left(\mathrm{RCA}_{0}\right)$ The following are equivalent:

(1) $\mathrm{ACA}_{0}$

(2) If $H$ is a hypergraph with finite edges represented by a sequence of edges, then $H$ can be represented by a set of edges. 
Proof. First we will prove that (11) implies (2). Reasoning in $\mathrm{ACA}_{0}$, let $H$ be a hypergraph with finite edges represented by the sequence $\left\langle e_{i}\right\rangle_{i \in \mathbb{N}}$. Arithmetical comprehension can prove the edge set $\left\{e \mid \exists i\left(e=e_{i}\right)\right\}$ exists.

To prove the converse, by Lemma III.1.3 of Simpson [7], it suffices to use item (2) to prove the existence of the range of an injection. Let $g: \mathbb{N} \rightarrow \mathbb{N}$ be an injection. Define the hypergraph $H=\langle V, E\rangle$ as follows. Let $\mathbb{N}$ be the set of vertices. Define a sequence of edges $\left\langle e_{n}\right\rangle_{n \in \mathbb{N}}$ by $e_{n}=\{0, g(n)+1\}$. Note that $m$ is in the range of $g$ if and only if the set $\{0, m+1\}$ is an edge of $H$. Given the set of edges of $H$, recursive comprehension proves the existence of the range of $g$.

Theorem 4. $\left(\mathrm{RCA}_{0}\right)$ The following are equivalent:

(1) $\mathrm{ACA}_{0}$

(2) If $H$ is a hypergraph with finite edges represented by a sequence of characteristic functions, then $H$ can be represented by a sequence of finite set codes for edges.

Proof. We begin by proving that 1 implies 2 , Reasoning in $\mathrm{ACA}_{0}$, let $H$ be a hypergraph with finite edges represented by the sequence of characteristic functions $\left\langle e_{i}\right\rangle_{i \in \mathbb{N}}$. Define $s_{i}=\left\{j \mid e_{i}(j)=1\right\}$ for $i \in \mathbb{N}$. Then the sequence $\left\langle s_{i}\right\rangle_{i \in \mathbb{N}}$ is arithmetically definable.

To prove the converse, it suffices to use statement 2 to prove the existence of a range of an injection. Let $g: \mathbb{N} \rightarrow \mathbb{N}$ be that injection. Let $H$ be the hypergraph with vertex set $\mathbb{N}$ and edges defined by the sequence of characteristic functions $\left\langle e_{i}\right\rangle_{i \in \mathbb{N}}$ defined as follows

$$
e_{i}(n)= \begin{cases}1 & n=2 i \vee n=2 i+2 \\ 0 & n=2 j \wedge j \notin\{i, i+1\} \\ 1 & n=2 j+1 \wedge g(j)=i \\ 0 & n=2 j+1 \wedge g(j) \neq i\end{cases}
$$

The recursive comprehension axiom proves the existence of $\left\langle e_{i}\right\rangle$ using $g$ as a parameter. Applying the principle of statement 2, let $\left\langle s_{i}\right\rangle_{i \in \mathbb{N}}$ be a sequence of finite set codes for the edges of $H$. Given any value $y$, successively examine the set codes until we locate the unique $s_{i}$ encoding a set containing $2 y$ and $2 y+2$. If this set contains only $2 y$ and $2 y+2$, then $y$ is not in the range of $g$, but if the set also contains another element, then $y$ is in the range of $g$. Thus, recursive comprehension proves the existence of the range of $g$. 
In order to state compactness results for vertex colorings, we need to consider finite substructures of hypergraphs. The following terminology is based on that of Berge [1]. When constructing substructures of a hypergraph $H$, one can either require that all edges in the substructure are edges of $H$, or allow edges of the substructure to be subsets of edges of $H$.

Definition. A partial hypergraph of a hypergraph $H$ consists of a subset $V$ of the vertices of $H$ and a subset $E$ of the edges of $H$ such that for every edge $e \in E, e \subset V$.

Definition. A partial subhypergraph of a hypergraph $H$ consists of a subset $V$ of the vertices of $H$ and a set $E$ of subsets of $V$ such that for every $e \in E$, there is an edge $e^{\prime} \in H$ such that $e=e^{\prime} \cap V$.

\section{Vertex colorings and finite edges}

A vertex coloring is a map from the vertices of a hypergraph into a set of colors, usually coded by a subset of $\mathbb{N}$. In the literature, most definitions for vertex colorings are stated for finite hypergraphs, but those listed below extend naturally to infinite hypergraphs. The definition for conflict-free colorings is based on that of Smorodinsky [8]. The other definitions can be found in the book of Berge [1].

Definition. Suppose $\langle V, E\rangle$ is a hypergraph. Let $\chi: V \rightarrow \mathbb{N}$ be a coloring of the vertices. We say:

(1) $\chi$ is a $k$-coloring if the range of $\chi$ is contained in $\{0,1, \ldots k-1\}$.

(2) $\chi$ is proper if $\chi$ is non-constant on each edge that contains more than one vertex.

(3) $\chi$ is strong if $\chi$ is injective on each edge.

(4) $\chi$ is conflict-free [8] if each edge contains one vertex whose color matches no other vertex in the edge. That is, $\forall e \exists j|\{v \in e \mid \chi(v)=j\}|=1$.

Theorem 3.4 of Hirst [4] states that $\mathrm{WKL}_{0}$ is equivalent to the statement that every locally $k$-colorable graph has a $k$-coloring. The following result generalizations this theorem to the hypergraph setting, for hypergraphs with sequences or sets of finite edges. 
Theorem 5. $\left(\mathrm{RCA}_{0}\right)$ For $k \geq 2$, the following are equivalent:

(1) $\mathrm{WKL}_{0}$.

(2) Let $H$ be a hypergraph with a sequence of finite edges. If every finite partial hypergraph of $H$ has a proper $k$-coloring, then $H$ has a proper $k$-coloring.

(3) Statement (2) with "proper" replaced by "conflict-free".

(4) Statement (2) with "sequence of edges" replaced by "set of edges."

(5) Statement (3) with "sequence of edges" replaced by "set of edges."

Proof. To prove that (1) implies (2), assume $\mathrm{WKL}_{0}$ and let $H$ be a hypergraph with vertex set $\left\{v_{1}, v_{2}, \ldots\right\}$ such that every finite partial hypergraph of $H$ has a proper $k$-coloring. For every $n$, let $m_{n}$ be the least integer such that $m_{n}>$ $m_{n-1}$ and if a vertex $v_{i}$ appears in one of the first $n$ edges of $H$ then $i \leq m_{n}$. Let $H_{n}$ be the finite partial hypergraph with vertex set $\left\{v_{1}, v_{2}, \ldots, v_{m_{n}}\right\}$ and the first $n$ edges of $H$. Let $T$ be the tree consisting of those finite sequences $\sigma$ in $k^{<\mathbb{N}}$ such that whenever length $(\sigma)=m_{n}, \sigma$ is a proper $k$-coloring of $H_{n}$. Since every finite partial hypergraph of $H$ has a proper $k$-coloring, $T$ must contain infinitely many sequences. By $\mathrm{WKL}_{0}, T$ has an infinite path. This path yields a proper $k$-coloring of $H$.

To prove that (11) implies (3), repeat the previous proof replacing every instance of the word "proper" by "conflict-free." Theorem 1 shows that (2) implies (4) and (3) implies (5) It remains to show that both (4) and (5) imply (11). For graphs, that is, for hypergraphs in which every edge has exactly two vertices, every coloring is proper if and only if it is conflict-free. Thus, both (44) and (5) imply that if every finite subgraph of a graph $H$ is $k$-colorable, then $H$ can be $k$-colored. This implies $\mathrm{WKL}_{0}$ by Theorem 3.4 of Hirst [4].

For the analog of the previous theorem for graphs with edges represented by sequences of characteristic functions, additional set comprehension strength is required.

Theorem 6. $\left(\mathrm{RCA}_{0}\right)$ For any $k \geq 2$, the following are equivalent.

(1) $\mathrm{ACA}_{0}$. 
(2) Suppose $H$ is a hypergraph with finite edges given by a sequence of characteristic functions. If every finite partial hypergraph of $H$ has a proper $k$-coloring then $H$ has a proper $k$-coloring.

(3) Statement (2) with "proper" replaced by "conflict-free."

Proof. To prove that (11) implies (2), fix $k$, assume $\mathrm{ACA}_{0}$, and suppose $H$ is a hypergraph with finite edges given by a sequence of characteristic functions. Further suppose that every finite partial hypergraph of $H$ can be properly $k$ colored. By Theorem 4, we can find the sequence of edges for $H$. By Theorem (5) and the fact that $\mathrm{ACA}_{0}$ implies $\mathrm{WKL}_{0}, H$ has a proper $k$-coloring.

The proof that (11) implies (3) is similar to the preceding argument, with proper replaced by conflict-free.

For the reversals, we begin by considering the proof that (2) implies (1) for $k=2$. By Lemma III.1.3 of Simpson [7], it suffices to use $\mathrm{RCA}_{0}$ and (2) to prove the existence of the range of an arbitrary injection $f$. The vertices of our hypergraph $H$ will be $\left\{b_{i} \mid i \in \mathbb{N}\right\} \cup\left\{v_{i, j} \mid i, j \in \mathbb{N}\right\}$. The edges of $H$ will be represented by characteristic functions for the following sets. For all $n$ and $i, p_{n, i}=\left\{v_{n, i}, v_{n, i+1}\right\}, q_{n}=\left\{b_{n}, b_{n+1}\right\}, r_{n}=\left\{b_{2 n}, v_{f(n), 2 n}\right\}$, and $s_{n}=\left\{v_{n, 0}, b_{2 n+1}\right\} \cup\left\{b_{2 i} \mid f(i)=n\right\}$. Because $f$ is an injection, $b_{2 i}$ is in $s_{n}$ if and only if $\exists t<2 i(f(t)=n)$, so the characteristic function for $s_{n}$ is uniformly computable from $f$. Via dovetailing, recursive comprehension suffices to prove the existence of a sequence of characteristic functions for all these edges of $H$. Note that edges of the form $s_{n}$ contain two vertices if $n$ is not in the range of $f$ and three vertices if $n$ is in the range. All other edges contain exactly two vertices.

We claim that every finite partial subhypergraph of $H$ has a proper 2coloring. Let $G$ be a partial subhypergraph. Let $n_{0}$ be the maximum natural number such that $b_{2 n_{0}}$ is in $G$. Define the coloring $\chi$ by $\chi\left(b_{n}\right)=n \bmod 2$ if $n \leq 2 n_{0}+1, \chi\left(v_{n, j}\right)=j \bmod 2$ if $\forall t \leq n_{0}(f(t) \neq n)$, and $\chi\left(v_{n, j}\right)=j+1$ mod 2 if $\exists t \leq n_{0}(f(t)=n)$. The first clause guarantees that $\chi$ is a proper coloring with regard to every edge of the form $q_{n}$, and the last two clauses insure that $\chi$ is proper on edges of the forms $p_{n, i}, r_{n}$ and $s_{n}$ restricted to the vertices of $G$.

Apply (2) to obtain a proper 2-coloring of $H$. If necessary, permute the colors so that $\chi\left(b_{0}\right)=0$. Fix $n$. If $f(t)=n$ for some $t$, then $r_{t}=\left\{b_{2 t}, v_{n, 2 t}\right\}$ is in $H$, so $\chi\left(v_{n, 0}\right)=1$. If $n$ is not in the range of $f$, then $s_{n}=\left\{v_{n, 0}, b_{2 n+1}\right\}$ is in $H$, so $\chi\left(v_{n, 0}\right)=0$. Thus the range of $f$ is defined by $\left\{n \mid \chi\left(v_{n, 0}\right)=1\right\}$, which exists by recursive comprehension. 
The proof that (2) implies (11) can be extended to values of $k$ greater than 2 by adding a complete graph on $k-2$ vertices to $H$ and connecting each vertex in the complete graph to every vertex in the prior construction. Finally, for graphs with edges of size at most 3, any coloring is conflict-free if and only if it is proper. Thus (3) implies (11) can be proved by replacing all uses of "proper" in the preceding arguments with "conflict-free."

For strong colorings, edge representation does not affect the strength of the coloring statements. Additionally, in some settings, finite colorability suffices to imply $\mathrm{WKL}_{0}$ over $\mathrm{RCA}_{0}$. This provides an interesting contrast to the case for graphs, as described following the proof of the next result.

Theorem 7. $\left(\mathrm{RCA}_{0}\right)$ The following are equivalent:

(1) $\mathrm{WKL}_{0}$.

(2) Let $H$ be a hypergraph with any edge representation. If there is a $k$ such that every finite partial hypergraph of $H$ has a strong $k$-coloring, then $H$ has a strong $k$-coloring.

(3) Let $H$ be a hypergraph with a set of finite sets for edges. If every finite partial hypergraph of $H$ has a strong 3 -coloring, then $H$ has a strong $k$-coloring for some $k$.

(4) Let $H$ be a hypergraph with a sequence of finite sets for edges. If every finite partial hypergraph of $H$ has a strong 2-coloring, then $H$ has a strong $k$-coloring for some $k$.

Proof. To prove that (1) implies (2), assume $\mathrm{WKL}_{0}$ and let $H$ be a hypergraph. By Theorem 1 and Theorem 2 , we may assume that the edges of $H$ are given by a sequence of characteristic functions. Fix $k$ and suppose that for every $n$, the partial subhypergraph given by the first $n$ values of each of the the first $n$ characteristic functions has a strong $k$-coloring. Let $T$ be the tree consisting of those finite sequences $\sigma$ in $k^{<\mathbb{N}}$ such that $\sigma$ is a strong $k$-coloring of the partial subhypergraph defined by the first length $(\sigma)$ many values of the first length $(\sigma)$ many edge characteristic functions. Because the finite partial hypergraphs of $H$ are colorable, $T$ must contain infinitely many sequences. By $\mathrm{WKL}_{0}, T$ has an infinite path. This path yields a strong $k$-coloring of $H$.

Note that (3) follows immediately from from (2) restricted to $k=3$. To prove that (3) implies (1), by Lemma IV.4.4 of Simpson [7], it suffices 
to use (3) to separate the ranges of injections with disjoint ranges. Fix injections $f$ and $g$ such that $\forall m \forall n(f(m) \neq g(n))$ and construct a hypergraph $H$ as follows. The vertices of $H$ consist of the sets $U=\left\{u_{i} \mid i \in \mathbb{N}\right\}$ and $V=\left\{v_{i} \mid i \in \mathbb{N}\right\}$. For each triple $i, j$, and $k$, the edge $\left\{u_{i}, u_{j}, v_{k}\right\}$ is in $H$ if and only if $i<k, j<k, \exists t<k f(t)=i$, and $\exists t<k g(t)=j$. Note that each edge contains exactly three vertices and exactly one vertex from $V$. No edge contains two vertices from $U$ indexed by elements of the range of $f$, and similarly, pairs of vertices from $U$ indexed by elements of the range of $g$ are forbidden within an edge. Let $H_{0}$ be a finite partial hypergraph consisting of vertices $\left\{u_{i_{0}}, \ldots u_{i_{m}}\right\}$ and $\left\{v_{j_{0}}, \ldots v_{j_{n}}\right\}$, and some or all of the edges of $H$ with vertices entirely contained in these sets. The coloring that assigns color 0 to all vertices $u_{i_{k}}$ such that $\exists t<j_{n} f(t)=i_{k}$, color 1 to all vertices $u_{i_{k}}$ such that $\exists t<j_{n} g(t)=i_{k}$, and color 3 to all remaining vertices is a strong 3-coloring of $H_{0}$. Thus, every finite partial hypergraph of $H$ has a strong 3coloring. Apply (3) to obtain a $k$-coloring of $H$. Note that if $\exists t f(t)=i$ and $\exists t g(t)=j$, then $u_{i}$ and $u_{j}$ are included in an edge, and so $u_{i}$ and $u_{j}$ must have distinct colors. By bounded $\Sigma_{1}^{0}$ comprehension (which is provable in $\mathrm{RCA}_{0}$ by Remark II.3.11 of Simpson [7]) the set $C=\left\{i<k \mid \exists t \chi\left(u_{f(t)}\right)=i\right\}$ exists. $\mathrm{RCA}_{0}$ proves that the set $\left\{j \in \mathbb{N} \mid \chi\left(u_{j}\right) \in C\right\}$ exists, contains the range of $f$, and is disjoint from the range of $g$.

Item (4) also follows immediately from (2). As in the previous paragraph, to prove that (4) implies $\mathrm{WKL}_{0}$, let $f$ and $g$ be injections with disjoint ranges. Construct $H$ as follows. The vertices of $H$ are $V=\left\{v_{i} \mid i \in \mathbb{N}\right\}$. Using a bijective pair encoding, for each $i \in \mathbb{N}$, let $i_{0}$ and $i_{1}$ denote the components of the pair encoded by $i$. For each $i$, define the edge $e_{i}=\left\{v_{f\left(i_{0}\right)}, v_{g\left(i_{1}\right)}\right\}$. Let $E_{0}$ be any finite set of edges with indices bounded by $b$, and let $V_{0}$ be some set of vertices containing all the vertices in edges in $E_{0}$. The coloring which assigns color 0 to all vertices of the form $v_{f(t)}$ for $t<b$ and color 1 to all other vertices of $V_{0}$ is a strong 2-coloring of the finite partial hypergraph defined by $E_{0}$ and $V_{0}$. By (4), $H$ has a strong $k$-coloring for some $k$; call it $\chi$. By bounded $\Sigma_{1}^{0}$ comprehension, provable in $\mathrm{RCA}_{0}$ by Exercise II.3.13 of Simpson [7], the set $K=\left\{j<k \mid \exists t \chi\left(v_{f(t)}\right)=j\right\}$ exists. By recursive comprehension, the set $S=\left\{n \mid \chi\left(v_{n}\right) \in K\right\}$ exists. By the construction of $H, S$ contains all elements of the range of $f$ and excludes all elements of the range of $g$.

In the preceding theorem, if the number 3 is replaced by 2 in item (3), the resulting statement asserts that every locally 2-colorable graph has a finite coloring. This statement is known to be equivalent to $\mathrm{WKL}_{0}$ over $\mathrm{RCA}_{0}$ plus 
$\Sigma_{2}^{0}$ induction, but the equivalence over $\mathrm{RCA}_{0}$ is one of many open questions listed in section $\S 5$ of Dorais, Hirst, and Shafer [3]. Item (4) shows that for graphs with edges presented as a sequence rather than as a set, the statement that every locally 2-colorable graph has a finite coloring is equivalent to $\mathrm{WKL}_{0}$ over RCA $\mathrm{R}_{0}$.

\section{Vertex colorings and infinite edges}

Allowing hypergraphs with infinite edges significantly affects the nature of vertex colorings. Clearly, any strong vertex coloring of a hypergraph with an infinite edge must use infinitely many colors. Hypergraphs with infinite edges may or may not have finite proper or conflict-free colorings. We will show that sorting those graphs with finite colorings from those without requires $\Pi_{1}^{1}-\mathrm{CA}_{0}$. The next definition and theorem assist in that proof.

Definition. If $T$ is a tree, the set $L$ of leaves of $T$ consists of those sequences in $T$ which have no extensions. That is, $L=\left\{\sigma \in T \mid \forall n \sigma^{\wedge} n \notin T\right\}$.

Lemma 8. $\left(\mathrm{RCA}_{0}\right)$ The follow are equivalent.

(1) $\Pi_{1}^{1}-\mathrm{CA}_{0}$.

(2) If $\left\langle T_{i}\right\rangle_{i \in \mathbb{N}}$ is a sequence of trees in $\mathbb{N}<\mathbb{N}$, then there is a function $f$ : $\mathbb{N} \rightarrow 2$ such that $f(i)=1$ if and only if $T_{i}$ contains an infinite path.

(3) If $\left\langle T_{i}\right\rangle_{i \in \mathbb{N}}$ is a sequence of trees and $\left\langle L_{i}\right\rangle_{i \in \mathbb{N}}$ is a sequence of sets such that for each $i, L_{i}$ is the set of leaves of $T_{i}$, then there is a function $f: \mathbb{N} \rightarrow 2$ such that $f(i)=1$ if and only if $T_{i}$ contains an infinite path.

Proof. We work in $\mathrm{RCA}_{0}$. The equivalence of (11) and (2) is Lemma VI.1.1 of Simpson [7]. Because (3) is a special case of (2), we need only prove that (3) implies (2).

Let $\left\langle T_{i}\right\rangle_{i \in \mathbb{N}}$ be a sequence of trees as in (2). For any sequence $\sigma$ of positive integers, let $\sigma_{-1}$ denote the sequence of the same length as $\sigma$ such that for all $i, \sigma_{-1}(i)=\sigma(i)-1$. For each $i$, define the tree $\hat{T}_{i}$ by letting $\tau \in \hat{T}_{i}$ if and only if either $\tau_{-1} \in T_{i}$ or $\tau=\sigma^{\frown} 0$ and $\sigma_{-1} \in T_{i}$. For each $i$, the leaf set of $\hat{T}_{i}$ consists precisely of those sequences of the form $\sigma_{-1}{ }^{\frown} 0$ such that $\sigma \in T_{i}$. $\mathrm{RCA}_{0}$ can prove that $\left\langle\hat{T}_{i}\right\rangle_{i \in \mathbb{N}}$ and $\left\langle L_{i}\right\rangle_{i \in \mathbb{N}}$ exist. Note that $p$ is an infinite path in $\hat{T}_{i}$ if and only if $p_{-1}$ is an infinite path in $T$. Thus, the function $f$ satisfying (3) for $\left\langle\hat{T}_{i}\right\rangle_{i \in \mathbb{N}}$ also satisfies (2) for $\left\langle T_{i}\right\rangle_{i \in \mathbb{N}}$. 
The preceding result allows us to prove the reversal below in a single step, rather than using (2) to prove $A C A_{0}$ and then deducing $\Pi_{1}^{1}-C_{0}$ in a second step.

Theorem 9. $\left(\mathrm{RCA}_{0}\right)$ For each $k \geq 2$, the following are equivalent.

(1) $\Pi_{1}^{1}-\mathrm{CA}_{0}$.

(2) If $\left\langle H_{i}\right\rangle_{i \in \mathbb{N}}$ is a sequence of hypergraphs, then there is a function $f$ : $\mathbb{N} \rightarrow 2$ such that $f(i)=1$ if and only if $H_{i}$ has a proper $k$-coloring.

(3) Statement (2) with "proper" replaced by "conflict-free."

Proof. Assume RCA $\mathrm{R}_{0}$. To prove that (11) implies (2), fix $k \geq 2$ and suppose $\left\langle H_{i}\right\rangle_{i \in \mathbb{N}}$ is a sequence of hypergraphs. Assuming that the vertices of $H$ are a subset of $\mathbb{N}$, the statement " $H$ has a proper $k$-coloring" asserts the existence of a function $g: \mathbb{N} \rightarrow k$ such that $g$ is not constant on any edge. Thus, the set of indices $i$ such that $H_{i}$ has a proper $k$-coloring is definable by a $\Sigma_{1}^{1}$ formula. Applying $\Pi_{1}^{1}-C_{0}$, the complement of this set of indices exists, so by recursive comprehension, the set of indices and its characteristic function also exist. This function satisfies item (2) of the theorem. A similar argument shows that (1) implies (3).

Next, we will prove that (2) implies (11) for the case $k=2$, indicating parenthetically how to modify the argument for (3) implies (11). By Lemma 8, it suffices to use (2) to determine which trees are well-founded in a list of trees with leaf sets. Let $\left\langle T_{i}\right\rangle_{i \in \mathbb{N}}$ be a sequence of trees in $\mathbb{N}<\mathbb{N}$, and let $\left\langle L_{i}\right\rangle_{i \in \mathbb{N}}$ be the corresponding leaf sets. Given any tree $T \subset \mathbb{N}^{\mathbb{N}}$ with leaf set $L$, define a hypergraph $H$ as follows. The vertices of $H$ are $\left\{a_{0}, a_{1}, b_{0}, b_{1}, s\right\}$ together with vertices labeled $\sigma_{0}$ and $\sigma_{1}$ for each nonempty sequence $\sigma$ in $T$. (One can routinely assign integer codes to these vertices, and arrange for the set of codes to be $\mathbb{N}$ or an initial segment of $\mathbb{N}$, if so desired.) The edges of $H$ consist of

- $\left(a_{0}, a_{1}\right),\left(a_{1}, s\right),\left(b_{0}, b_{1}\right)$, and $\left(b_{1}, s\right)$,

- $\left(\sigma_{0}, \sigma_{1}\right)$ for every nonempty $\sigma \in T$,

- $\left(\sigma_{1}, s\right)$ if $\sigma$ is a leaf of $T$,

- $E_{\sigma}=\left\{\sigma_{1}\right\} \cup\left\{\tau_{0} \mid \tau \in T \wedge \exists n \tau=\sigma^{\wedge} n\right\}$ if $\sigma \in T$ is not a leaf, and 


$$
\text { - } E_{0}=\left\{a_{0}, b_{0}\right\} \cup\left\{\sigma_{0} \mid \sigma \in T \wedge \operatorname{length}(\sigma)=1\right\} \text {. }
$$

Using $T$ and $L$ as parameters, RCA $_{0}$ can prove the existence of $H$ uniformly. Note that $E_{0}$ and $E_{\sigma}$ may be infinite edges.

Suppose $c: \mathbb{N} \rightarrow$ red, blue $\}$ is any 2-coloring of the vertices of $H$. Swapping colors if necessary, let $s$ be blue. Assume $c$ is proper. Then $a_{1}$ and $b_{1}$ are red and $a_{0}$ and $b_{0}$ are blue. Also, for any $\sigma \in T$ if $\sigma_{0}$ is red, then $\sigma_{1}$ is blue, so $\left\{\sigma_{1}, s\right\}$ is not an edge and $\sigma$ is not a leaf. Because $\sigma_{1}$ is blue, by the definition of $E_{\sigma}$, for some immediate successor $\tau$ of $\sigma, \tau_{0}$ must be red. Finally, to properly color $E_{0}$, for some $\sigma \in T$ of length $1, \sigma_{0}$ is red. Let $n$ be the least value such that for $\tau=\sigma^{\wedge} n, \tau_{0}$ is red. Iterating this process traces an infinite path in $T$. Summarizing, if $c$ properly 2-colors $H$, then $T$ has an infinite path. (Every conflict-free coloring is proper, so if $c$ is conflict-free then $T$ has an infinite path.)

Conversely, suppose that $T$ has an infinite path $p$, and let $\sigma^{0} \subset \sigma^{1} \subset$ $\sigma^{2} \subset \ldots$ be the nonempty initial segments of $p$. Define $c$ by $c(s)=c\left(a_{0}\right)=$ $c\left(b_{0}\right)=$ blue, $c\left(a_{1}\right)=c\left(b_{1}\right)=$ red, $\sigma_{0}^{i}=\operatorname{red}$ and $\sigma_{1}^{1}=$ blue for $\sigma^{i}$ in $p$, and $\tau_{0}=$ blue and $\tau_{1}=$ red for $\tau \in T$ not in $p$. Treating the definitions of the edges of $H$ as cases, one can verify that $c$ is a proper (conflict-free) 2 coloring of $H$. Summarizing the last two paragraphs, $H$ has a proper (conflict-free) 2-coloring if and only if $T$ has an infinite path.

Carrying out the the construction uniformly for all the trees in $\left\langle T_{i}\right\rangle_{i \in \mathbb{N}}$ and applying (2) of the theorem, we can find the characteristic function for the well-founded trees, as desired. The parenthetical comments show that for $k=2$, (3) implies (1).

For values of $k>2$, modify the construction of the previous reversal by adding a complete graph with $k-2$ vertices to $H$, connecting each vertex of the complete graph to every vertex of $H$ by an edge consisting of two vertices. The resulting hypergraph has a proper (conflict-free) $k$-coloring if and only if $T$ is not well-founded.

It would be interesting to know if the preceding result continues to hold if " $k$-coloring" is replaced by "finite coloring." Lemma 8 may prove useful in recasting the preceding theorem as a result related to $\Sigma_{1}^{1}$ completness using many-one reducibility, or as a result on Weihrauch reducibility.

Infinite edges can interfere with finite conflict-free colorings of hypergraphs. The following graph illustrates this situation. 
Definition. The $\mathcal{M}$-graph (the Matryoshka graph) is the hypergraph with vertex set $\mathbb{N}$ and edges $\left\{E_{j} \mid j \in \mathbb{N}\right\}$ where $E_{j}=\{k \mid j \leq k\}$.

Every finite partial subhypergraph of the $\mathcal{M}$-graph has a conflict-free 2coloring. For example, given any finite collection of vertices, simply color the largest numbered vertex red and all other vertices blue. On the other hand, the entire $\mathcal{M}$-graph has no finite conflict-free coloring. This can be proved directly by induction on $\Sigma_{2}^{0}$ formulas, or by using the following lemma.

Lemma 10. $\left(\mathrm{RCA}_{0}\right)$ The following are equivalent.

(1) ERT (eventually repeating tails): Suppose $f: \mathbb{N} \rightarrow k$ for some $k \in \mathbb{N}$. Then there is $a b \in \mathbb{N}$ such that for all $x \geq b$ there is $a y \geq b$ such that $x \neq y$ and $f(x)=f(y)$.

(2) No finite coloring of the $\mathcal{M}$-graph is conflict-free.

Proof. We will work in $\mathrm{RCA}_{0}$. To prove that (1) implies (2), suppose that $f: \mathbb{N} \rightarrow k$ is a finite coloring of the $\mathcal{M}$-graph. Apply ERT to find $b$ such that for all $x \geq b$ there is a $y \geq b$ such that $y \neq x$ and $f(x)=f(y)$. Then every color appearing in $E_{b}$ appears at least twice. Thus $f$ is not conflict-free.

To prove the converse, let $f: \mathbb{N} \rightarrow k$ be any function. We can view $f$ as a $k$-coloring of the $\mathcal{M}$-graph. By (2),$f$ is not conflict-free. Thus there is an edge $E_{b}$ such that every color appearing in $b$ appears at least twice. Thus $b$ witnesses that ERT holds.

The principle ERT follows trivially from the principle ECT which asserts that if $f: \mathbb{N} \rightarrow k$ then there is a $b$ such that for all $x \geq b$ there are infinitely many values of $y$ such that $f(x)=f(y)$. By Theorem 6 of Hirst [5], ECT is equivalent to $\Sigma_{2}^{0}$ induction. Thus, $I \Sigma_{2}^{0}$ suffices to prove that no finite coloring of the $\mathcal{M}$-graph is conflict-free.

We will show that this use of induction is not necessary by proving ERT from $\mathrm{SRT}_{2}^{2}$, the stable Ramsey theorem for pairs and two colors. By Corollary 2.6 of Chong, Slaman, and Yang [2], $\mathrm{SRT}_{2}^{2}$ cannot prove $\mathrm{I}_{2}^{0}$, so neither can ERT.

Theorem 11. $\left(\mathrm{RCA}_{0}\right) \mathrm{SRT}_{2}^{2}$ implies ERT.

Proof. Let $f: \mathbb{N} \rightarrow k$. Define $g:[\mathbb{N}]^{2} \rightarrow 2$ by $g(a, b)=1$ if and only if for some $x \in[a, b), f(x)$ appears exactly once in the range of $f$ restricted to 
$[a, b)$. Because the range of $f$ is $k$, for fixed $a$ and increasing $x$, the value of $g(a, x)$ can only change at most $2 k$ times. Thus $g$ is a stable coloring.

Apply SRT 2 to $g$ to obtain an infinite set $H=\left\{x_{0}, x_{1}, x_{2} \ldots\right\}$ that is monochromatic for $g$. Suppose, by way of contradiction, that $g\left([H]^{2}\right) \equiv 1$. Let $H_{0}$ consist of the first $3 \cdot 2^{k-1}$ elements of $H$. The elements of $H_{0}$ define $3 \cdot 2^{k-1}-1$ consecutive half-open intervals. Because $g\left(x_{0}, x_{3 \cdot 2^{k-1}}\right)=1$, some value of $f$ appears exactly once in $\left[x_{0}, x_{3 \cdot 2^{k-1}}\right)$. Call this $k_{0}$. Either on the left or the right of this location, there must be a collection of $3 \cdot 2^{k-2}$ consecutive elements of $H_{0}$. Call these $H_{1}$. Note that the range of $f$ on the intervals defined by $H_{1}$ must omit $k_{0}$. Thus, the range of $f$ on these intervals contains at most $k-1$ elements. Iterating this construction, $H_{k-1}$ will consist of three consecutive elements of $H_{0}$ such that $f$ is constant on the union of the two associated subintervals. No value of $f$ appears exactly once in the range of $f$ on this union, contradicting the assumption that $g$ applied to the endpoints yields 1 . Thus $g\left([H]^{2}\right) \equiv 0$ and every value of $f$ appearing at or after $x_{0}$ must appear at least twice.

It would be interesting to know how ERT compares in strength to other statements that are weaker than $I \Sigma_{2}^{0}$, for example, like those described by Kreuzer and Yokoyama [6]

\section{Bibliography}

[1] Claude Berge, Hypergraphs, North-Holland Mathematical Library, vol. 45, NorthHolland Publishing Co., Amsterdam, 1989. Combinatorics of finite sets; Translated from the French. MR1013569

[2] C. T. Chong, Theodore A. Slaman, and Yue Yang, The metamathematics of stable Ramsey's theorem for pairs, J. Amer. Math. Soc. 27 (2014), no. 3, 863-892. MR3194495

[3] François G. Dorais, Jeffry L. Hirst, and Paul Shafer, Comparing the strength of diagonally nonrecursive functions in the absence of $\Sigma_{2}^{0}$ induction, J. Symb. Log. 80 (2015), no. 4, 1211-1235. MR3436365

[4] Jeffry L. Hirst, Marriage theorems and reverse mathematics, Logic and computation (Pittsburgh, PA, 1987), Contemp. Math., vol. 106, Amer. Math. Soc., Providence, RI, 1990, pp. 181-196, DOI 10.1090/conm/106/1057822. MR1057822

[5] _ Disguising induction: proofs of the pigeonhole principle for trees, Foundational adventures, Tributes, vol. 22, Coll. Publ., London, 2014, pp. 113-123. MR3241956 
[6] Alexander P. Kreuzer and Keita Yokoyama, On principles between $\Sigma_{1}$ - and $\Sigma_{2}$ induction, and monotone enumerations, J. Math. Log. 16 (2016), no. 1, 1650004, 21. MR3518781

[7] Stephen G. Simpson, Subsystems of second order arithmetic, 2nd ed., Perspectives in Logic, Cambridge University Press, Cambridge; Association for Symbolic Logic, Poughkeepsie, NY, 2009. MR2517689

[8] Shakhar Smorodinsky, Conflict-free coloring and its applications, Geometry-intuitive, discrete, and convex, Bolyai Soc. Math. Stud., vol. 24, János Bolyai Math. Soc., Budapest, 2013, pp. 331-389, DOI 10.1007/978-3-642-41498-5_12. MR3204564 